\title{
Uniform Estimates of Elementary Solutions of First Order Systems of Partial Differential Equations
}

\author{
By \\ Mutsuhide Matsumura*
}

\section{Introduction}

The asymptotic behavior for $|x| \rightarrow \infty$ of solutions of partial differential equations with constant coefficients has been studied by V. V. Grušin [1], W. Littman [3], B. R. Vainberg [8], etc (see also C. H. Wilcox [9] and J.R. Schulenberger $[\boldsymbol{7}]$ ). This paper is concerned with the asymptotic behavior of elementary solutions for a certain class of first order systems with constant coefficients. We shall give some estimates which are uniform with respect to a complex parameter taking on values in a certain region of the complex plane. ${ }^{1)}$

Assumptions and results. The systems which we shall consider are of the form

$$
\left(\frac{1}{i} \sum_{j=1}^{n} A_{j} \frac{\partial}{\partial x_{j}}-\lambda I\right) v(x)=g(x), \quad x \in \boldsymbol{R}^{n} .
$$

Here the $A_{j}$ are $N \times N$ constant matrices, $I$ is the unit matrix of order $N, \lambda$ is a complex parameter, and $v(x)$ and $g(x)$ are column vectors with $N$ components. The characteristic polynomial associated with (1) is

$$
\Delta(\xi ; \lambda)=\operatorname{det}\left(\lambda I-\sum_{j=1}^{n} \xi_{j} A_{j}\right)
$$

Received April 11, 1970.

Communicated by S. Matsuura.

* Department of Applied Mathematics and Physics, Faculty of Engineering, Kyoto University.

1) The author already remarked in [5] that the results of this paper can be proved by the same method as for the isotropic case in [4]. 
where $\xi=\left(\xi_{1}, \ldots, \xi_{n}\right)$ denotes a generic point of the real dual space $\xi^{n}$ of the $n$-dimensional euclidean space $\boldsymbol{R}^{n}$.

Now we state precisely the assumptions that we impose on the operator $A-\lambda I=\frac{1}{i} \sum_{j=1}^{n} A_{j} \frac{\partial}{\partial x_{j}}-\lambda I$.

i) The $N$ roots $\lambda_{k}(\xi)$ of $\Delta(\xi ; \lambda)=0$ are real and have constant multiplicity for all real $\xi \neq 0$ : i.e.,

$$
\Delta(\xi ; \lambda)=\prod_{k=1}^{m}\left(\lambda-\lambda_{k}(\xi)\right)^{\alpha_{k}}, \alpha_{1}+\cdots+\alpha_{m}=N
$$

where we label $\lambda_{k}(\xi)$ in decreasing order:

$$
\lambda_{1}(\xi)>\lambda_{2}(\xi)>\cdots>\lambda_{m}(\xi)
$$

for all real $\xi \neq 0$.

ii) A root $\lambda_{k}(\xi)$ vanishes for some real $\xi \neq 0$ only if it is identically zero.

iii) The matrix $A(\xi)=\sum_{j=1}^{n} \xi_{j} A_{j}$ is diagonalizable for any $\xi \neq 0 .{ }^{2)}$

iv) The $[m / 2]$ normal surfaces $S_{k}=\left\{\xi ; \lambda_{k}(\xi)=1\right\}, k=1, \ldots,[m / 2]^{3)}$ are non-singular and their Gaussian or total curvatures $K_{k}(\xi)$ do not vanish anywhere. Further, for any unit vector $\theta$ there exists only a finite number of points on $S_{k}$ at which the normal to $S_{k}$ is parallel to $\theta$.

From i), the matrix $A(\xi)-\lambda I$ is non-singular for each non-real $\lambda$. Hence $(A(\xi)-\lambda I)^{-1}$ has the inverse Fourier transform

$$
E(x ; \lambda)=(2 \pi)^{-n} \int_{\mathbb{\Omega}^{n}} \exp \{i\langle x, \xi\rangle\}(A(\xi)-\lambda I)^{-1} d \xi
$$

in the distribution sense. $E(x ; \lambda)$ is an elementary solution of the differential operator $A-\lambda I$ with non real $\lambda:(A-\lambda I) E(x ; \lambda)=\delta(x) I$ where $\delta(x)$ is the Dirac $\delta$-distribution. Note that this elementary solution $E(x ; \lambda)$ is the resolvent kernel of the operator $A-\lambda I$ in $L^{2}\left(\boldsymbol{R}^{n}\right)$ with domain

2) If the matrices $A_{j}, j=1, \cdots, m$ are hermitian, a simple criterion which ensures the conditions i) and ii) ( iii) is automatically satisfied) is found by Wilcox [9]. $\mathrm{He}$ calls such systems "uniformly propagative".

3) If $l$ is a real number, $[l]$ denotes the greatest integer not exceeding $l$. See also the relation (28). 


$$
D(A-\lambda I)=\left\{v ; v \text { and }(A-\lambda I) v \text { are in } L^{2}\left(\boldsymbol{R}^{n}\right)\right\}
$$

Our aim is to establish the following theorem:

Theorem. Under the assumptions (i)-(iv) the elementary solution $E(x ; \lambda)$ has the following properties:

$1^{\circ} \quad E(x ; \lambda)$ is an analytic function of $(x, \lambda)$ in $\left(\mathbb{R}^{n}-\{0\}\right) \times(\mathbb{C}-\mathbb{R})$.

$2^{\circ}$ For every multi-index $\nu$ and for every $(x, \sigma) \in\left(\boldsymbol{R}^{n}-\{0\}\right) \times(\boldsymbol{R}$ $-\{0\})$, the limits

$$
E^{(\nu)}(x ; \sigma \pm i 0)=\lim _{\varepsilon \rightarrow 0+}\left(\frac{\partial}{\partial x}\right)^{\nu} E(x ; \sigma \pm i \varepsilon)
$$

exist, where the convergence is uniform in every compact set of $\left(\mathbb{R}^{n}-\{0\}\right)$. Furthermore $E^{(\nu)}(x ; \sigma \pm i 0)$ are continuous functions of $(x, \sigma)$ in $\left(\boldsymbol{R}^{n}-\{0\}\right)$ $\times(\boldsymbol{R}-\{0\})$.

$3^{\circ}$ Let $\sigma^{\circ}$ be an arbitrary fixed real number and $\neq 0$. If we choose $\delta>0$ and $\varepsilon^{0}>0$ sufficiently small, then $E^{(\nu)}(x ; \sigma \pm i \varepsilon)$ behave for $\sigma \in\left(\sigma^{0}-\delta\right.$, $\left.\sigma^{0}+\delta\right)$ and $\varepsilon \in\left[0, \varepsilon^{0}\right)$ like

$$
E^{(\nu)}(x ; \sigma \pm i \varepsilon)=e_{\nu}^{ \pm}(x, \sigma, \varepsilon)|x|^{-(n-1) / 2}+O\left(|x|^{-n / 2}\right)
$$

as $|x| \rightarrow \infty$, where $e_{\nu}^{ \pm}(x, \sigma, \varepsilon)^{4)}$ are bounded continuous functions of $(x, \sigma, \varepsilon)$ in $\left\{(x, \sigma, \varepsilon) ;|x| \geqslant 1,\left|\sigma-\sigma^{0}\right|<\delta, 0 \leqslant \varepsilon<\varepsilon^{0}\right\}$.

$4^{\circ} \quad E^{(\nu)}(x ; \sigma \pm i \varepsilon)$ are Holder continuous in $\sigma$, and we have the following estimates uniformly in $\sigma, \sigma^{\prime} \in\left(\sigma^{0}-\delta, \sigma^{0}+\delta\right)$ and $\varepsilon \in\left[0, \varepsilon^{0}\right)$.

$$
\left|E^{(\nu)}(x ; \sigma \pm i \varepsilon)-E^{(\nu)}\left(x ; \sigma^{\prime} \pm i \varepsilon\right)\right| \leqslant \mathrm{const}|x|^{-(n-3) / 2}\left|\sigma-\sigma^{\prime}\right|^{\gamma}
$$

where $\gamma$ is any constant such that $0<\gamma<1$, and the 'const' is independent of $x(|x| \geqslant 1), \varepsilon, \sigma$ and $\sigma^{\prime}$.

The property $1^{\circ}$ is well-known. $2^{\circ}$ and $3^{\circ}$ are proved in $[4]$ and $4^{\circ}$ in Mochizuki $[6]$ for the isotropic case: $\lambda_{k}(\xi)=\tau_{k}|\xi|, k=1, \ldots, m$. Our proof for this general case can be given along same lines as for the isotropic case. ${ }^{5)}$

4) As to the explicit formulas, see the formulas (38)-(40).

5) Wilcox [9] also proved $2^{\circ}$ and partially $3^{\circ}$ (non uniform estimate). His method is to reduce the problem to higher order single operator's one (Grušin-LittmanVainberg case). The author's idea in [4] is to reduce the problem to one dimensional problem by making use of spectral representation of matrices. 


\section{Some Lemmas}

The following lemma is concerned with the behavior at infinity of Fourier transform of a smooth mass density concentrated on a surface, which has been investigated by Grušin $[\mathbf{1}]$, Littman [2], Vainberg [8], etc.

Lemma 1. Let $S$ be a $C^{\infty}$ closed $^{6)}$ non-singular surface of dimension $n-1$ in $\Xi^{n}$, $\mu$ a $C^{\infty}$ function on $S$. Assume that at each point $s$ of $S$ the Gaussian curvature $K(s)$ is different from zero, and that for each unit vector $\theta$, there exists only a finite set of points (denoted by $s^{l}=s^{l}(\theta)$ ) on $S$ at which the normal to $S$ is parallel to $\theta$. Taking $\theta$ as the positive direction at $s^{l}(\theta)$, denote by $p_{l}^{+} \equiv p_{l}^{+}(\theta)$ and $p_{l}^{-} \equiv p_{l}^{-}(\theta)$ the number of positive and negative principal curvatures at $s^{l}(\theta)$ respectively. Then the following asymptotic formula holds as $|x| \rightarrow \infty$, and $x /|x|=\theta$, uniformly with respect to all directions $\theta$.

$$
\begin{aligned}
& \int_{S} \mu(s) \exp \{i<x, s>\} d S \\
& \quad=\sum_{l} C_{l}(\theta) \exp \left\{i|x|<\theta, s^{l}(\theta)>\right\} \cdot|x|^{-(n-1) / 2}+q(x)
\end{aligned}
$$

where $d S$ is the surface element on $S$, and

$$
\begin{aligned}
C_{l}(\theta) & =(2 \pi)^{(n-1) / 2} \exp \left\{i \frac{\pi}{4}\left(p_{l}^{+}(\theta)-p_{l}^{-}(\theta)\right\} \frac{\mu\left(s^{l}(\theta)\right)}{\sqrt{\left|K\left(s^{l}(\theta)\right)\right|}}\right. \\
& =\pi^{(n-1) / 2}(1+i)^{p_{l}^{+}(\theta)}(1-i)^{p_{l}^{-}(\theta)} \frac{\mu\left(s^{l}(\theta)\right)}{\sqrt{\left|K\left(s^{l}(\theta)\right)\right|}}
\end{aligned}
$$

and $q(x)$ is estimated for large $|x|$ as

$$
|q(x)|+\sum_{j=1}^{n}\left|\frac{\partial q}{\partial x_{j}}(x)\right| \leqslant \text { const }|x|^{-(n+1) / 2} .
$$

For a proof of this lemma, see $[2]$. When $S$ is a sphere, an asymptotic formula with estimate (11) is given in Appendix of [4]. We can show (9)-(11) for the general case in a similar way.

6) $S$ need not be a closed surface. If $S$ has a boundary, we assume that $S$ does not contain its boundary and that $\mu$ has compact support in $S$. 
Let $\lambda(\xi)$ be a $C^{\infty}$ real-valued function defined in $g^{n}-\{0\}$ with the properties

(i) $\lambda(\xi)$ is positively homogeneous of degree 1 .

(ii) $\lambda(\xi)>0$ for all $\xi \neq 0$.

Then the set $S=\left\{s ; \lambda(s)=1, s \in \boldsymbol{\Xi}^{n}\right\}$ forms a $C^{\infty}$ closed non-singular ${ }^{7)}$ surface of dimension $n-1$ which encloses the origin. Let us suppose that the Gaussian curvature $K(s)$ does not vanish on $S$ and that, given a unit vector $\theta$, there is only a finite number of points (denoted by $s^{l}=s^{l}(\theta)$ on $S$ such that normal to $S$ at this point is parallel to $\theta$. Let $\sigma^{0}$ be an arbitrary fixed positive number. Let $U$ and $V$ be two relatively compact open neighborhoods of $\sigma^{0} S=\left\{\sigma^{0} s ; s \in S\right\}$ such that the closure of $U$ is contained in $V$ and $0 \notin V$. Let $\phi \in C_{0}^{\infty}(V)$ be equal to 1 in $U$ and $P$ a $C^{\infty}$ function defined in $g^{n}-\{0\}$ which is positively homogeneous of degree d. We shall study the asymptotic behavior as $|x| \rightarrow \infty$ of the function given by

$$
\Phi(x ; \sigma \pm i \varepsilon)=\int_{\Xi^{n}} \frac{\exp \{i<x, \xi>\} P(\xi)}{\lambda(\xi)-(\sigma \pm i \varepsilon)} \phi(\xi) d \xi
$$

where $\sigma \in\left(\sigma^{0}-\delta, \sigma^{0}+\delta\right)$ and $\varepsilon \in\left[0, \varepsilon^{0}\right)$.

Using the above function $\lambda(\xi)$, we can introduce new coordinates $(\rho, s)$ in the space $\xi^{n}-\{0\}$ such that $\xi=\rho s, 0<\rho<\infty$ and $s \in S$. Then we have

$$
d \xi_{1} \ldots d \xi_{n}=\left(\rho^{n-1} /|\operatorname{grad} \lambda(s)|\right) d \rho d S .
$$

In fact, from the assumption i) we may assume $\frac{\partial \lambda}{\partial s_{n}}(s) \neq 0$ in a small neighborhood of a point $s^{0}$ on $S$ without loss of generality. ${ }^{8}$ Then there exists a $C^{\infty}$ function $s_{n}=h\left(s_{1}, \ldots, s_{n-1}\right)$ such that $\lambda\left(s_{1}, \ldots, s_{n-1}\right.$, $\left.h\left(s_{1}, \ldots, s_{n-1}\right)\right) \equiv 0$ (in a small neighborhood of $\left.\left(s_{1}^{0} \ldots s_{n-1}^{0}\right)\right)$ and $\frac{\partial h}{\partial s_{j}}$ $=-\frac{\partial \lambda}{\partial s_{j}} / \frac{\partial \lambda}{\partial s_{n}}, j=1, \ldots, n-1$. Consequently we have

7) See the footnote 8).

8) Note that from i), ii) and Euler's relation $\sum_{j=1}^{n} \xi_{j} \frac{\partial \lambda}{\partial \xi_{j}}(\xi)=\lambda_{j}(\xi)$, we have $\operatorname{grad} \lambda(\xi)$ $\neq 0$ for $\xi \neq 0$. 


$$
\begin{aligned}
& \frac{D\left(\xi_{1}, \xi_{2}, \ldots, \xi_{n}\right)}{D\left(\rho, s_{1}, \ldots, s_{n-1}\right)}=(-1)^{n} \rho^{n-1}\left(\sum_{j=1}^{n-1} s_{j} \frac{\partial h}{\partial s_{j}}-h\right) \\
& \quad=(-1)^{n+1} \rho^{n-1}\left(\sum_{j=1}^{n} s_{j} \frac{\partial \lambda}{\partial s_{j}}\right) / \frac{\partial \lambda}{\partial s_{n}}=(-1)^{n-1} \rho^{n-1} / \frac{\partial \lambda}{\partial s_{n}}
\end{aligned}
$$

because $\sum_{j=1}^{n} s_{j} \frac{\partial \lambda}{\partial s_{j}}(s)=\lambda(s)=1$ for $s \in S$. Thus we get (9) from (10) and from a well known relation

$$
d S=\left(\sqrt{\sum_{j=1}^{n}\left(\frac{\partial \lambda}{\partial s_{j}}\right)^{2}} /\left|\frac{\partial \lambda}{\partial s_{n}}\right|\right) d s_{1} \ldots d s_{n-1} .
$$

Let $\psi \in C_{0}^{\infty}\left(\boldsymbol{R}^{1}\right)$ and satisfy $\operatorname{supp} \psi C\left\{\rho ;\left|\rho-\sigma^{0}\right|<4 \delta\right\}, \psi(\rho)=1$ for $\left|\rho-\sigma^{0}\right|<3 \delta$. Then $\phi(\rho)=\chi(\rho s)=\chi(\xi)$ defines a function in $\boldsymbol{g}^{n}-\{0\}$ and $\chi \in C_{0}^{\infty}\left(g^{n}-\{0\}\right), \operatorname{supp} \chi \subset V$ if we choose $\delta>0$ small, and $\chi(\xi)=1$ in a neighborhood of $S$. Using the function $\chi(\xi)$, we write $\Phi(x ; \sigma \pm i \varepsilon)$ in the form

$$
\begin{aligned}
& \Phi(x ; \sigma \pm i \varepsilon)=\int_{\nabla^{n}} \frac{\exp \{i<x, \xi>\} P(\xi)}{\lambda(\xi)-(\sigma \pm i \varepsilon)}(\phi(\xi)-\chi(\xi)) d \xi \\
& \quad+\int_{\Xi^{n}} \frac{\exp \{i<x, \xi>\} P(\xi)}{\lambda(\xi)-(\sigma \pm i \varepsilon)} \chi(\xi) d \xi \equiv \Phi^{(1)}(x ; \sigma \pm i \varepsilon)+\Phi^{(2)}(x ; \sigma \pm i \varepsilon) .
\end{aligned}
$$

First, we examine $\Phi^{(1)}(x ; \lambda)$ where $\lambda=\sigma \pm i \varepsilon$. Since $P(\xi)(\phi(\xi)-\chi(\xi))$ $/(\lambda(\xi)-\lambda)$ is a $C^{\infty}$ function of $\xi$ with compact support for each $\lambda$ in $\Lambda=\left\{\lambda ;\left|\operatorname{Re} \lambda-\sigma^{0}\right|<2 \delta\right\}$ and analytic with respect to $\lambda$ in $\Lambda$, it follows that $\Phi^{(1)}(x ; \lambda)$ is $C^{\infty}$ in $x$ and analytic in $\lambda$, and further that for any multi-index $\nu$ and any integer $p$, there exists a constant $C_{p, \nu}$ independent of $\lambda \in\left\{\lambda=\sigma \pm i \varepsilon ;\left|\sigma-\sigma^{0}\right|<\delta, 0 \leqslant \varepsilon<\varepsilon^{0}\right\}$ such that

$$
(1+|x|)^{p}\left|\left(\frac{\partial}{\partial \lambda}\right)\left(\frac{\partial}{\partial x}\right)^{\nu} \Phi^{(1)}(x ; \lambda)\right| \leqslant C_{p, \nu} .
$$

Now we consider $\left(\frac{\partial}{\partial x}\right)^{\nu} \Phi^{(2)}(x ; \sigma \pm i \varepsilon)$. If we use the new coordinates $(\rho, s)$ in $g^{n}$ which we have introduced above, we find for $\varepsilon>0$

$$
\left(\frac{\partial}{\partial x}\right)^{\nu} \Phi^{(2)}(x ; \sigma \pm i \varepsilon)
$$




$$
=\int_{0}^{\infty} \frac{\rho^{d+(n-1)+|\nu|} \phi(\rho)}{\rho-(\sigma \pm i \varepsilon)}\left\{\int_{S} \exp \{i \rho<x, s>\} \frac{(i s)^{\nu} P(s)}{|\operatorname{grad} \lambda(s)|} d S\right\} d \rho
$$

Replacing the interval $\left[\sigma^{0}-2 \delta, \sigma^{0}+2 \delta\right]$ in the contour $[0, \infty)$ of $(16)$ by a semi-circle in the lower or upper half-plane according as $\sigma+i \varepsilon$ or $\sigma-i \varepsilon$, we see that the limits $\left(\frac{\partial}{\partial x}\right)^{\nu} \Phi^{(2)}(x ; \sigma \pm i 0)$ exist and define continuous functions of $(x, \sigma)$.

If we now apply Lemma 1 to the integral in brackets of (16), we obtain

$$
\begin{aligned}
& \left(\frac{\partial}{\partial x}\right)^{\nu} \Phi^{(2)}(x ; \sigma \pm i \varepsilon)=\sum_{l} \exp \left\{i \frac{\pi}{4}\left(p_{l}^{+}(\theta)-p_{l}^{-}(\theta)\right)\right\} \\
& \quad \times \frac{\left(i s^{l}(\theta)\right)^{\nu} P\left(s^{l}(\theta)\right)}{\sqrt{\left|K\left(s^{l}(\theta)\right)\right|} \cdot\left|\operatorname{grad} \lambda\left(s^{l}(\theta)\right)\right|} \\
& \quad \times J_{l}^{ \pm}(|x|, \theta, \sigma, \varepsilon)|x|^{-(n-1) / 2}+\int_{0}^{\infty} \frac{\varphi_{1}(\rho) q_{\nu}(\rho x)}{\rho-(\sigma \pm i \varepsilon)} d \rho,
\end{aligned}
$$

where we have set

$$
J_{l}^{ \pm}(|x|, \theta, \sigma, \varepsilon)=(2 \pi)^{(n-1) / 2} \int_{0}^{\infty} \exp \left\{i<\theta, s^{l}(\theta)>\rho|x|\right\} \frac{\varphi(\rho)}{\rho-(\sigma \pm i \varepsilon)} d \rho
$$

and

$$
\varphi_{1}(\rho)=\rho^{d+|\nu|+(n-1)} \psi(\rho) \in C_{0}^{\infty}\left(\sigma^{0}-4 \delta, \sigma^{0}+4 \delta\right), \varphi(\rho)=\rho^{-(n-1) / 2} \varphi_{1}(\rho) .
$$

Then, from the relations

$$
\int_{-\infty}^{+\infty} \frac{\exp \{i \rho t\}}{\rho-(\sigma \pm i \varepsilon)} d \rho= \pm 2 \pi i Y( \pm t) \exp \{i(\sigma \pm i \varepsilon) t\}
$$

( $Y(t)$ is the Heaviside function), we find

$$
\begin{aligned}
J^{ \pm}(|x|, \theta, \sigma, \varepsilon)= & \pm(2 \pi)^{(n+1) / 2} \int_{-\infty}^{\infty} Y\left( \pm\left\{<\theta, s^{l}(\theta)>|x|-\tau\right\}\right) \\
& \times \exp \left\{i(\sigma \pm i \varepsilon)\left(<\theta, s^{l}(\theta)>|x|-\tau\right)\right\} \tilde{\varphi}(\tau) d \tau
\end{aligned}
$$

where $\tilde{\varphi}(\tau)=\int_{-\infty}^{+\infty} \exp \{i \rho \tau\} \varphi(\rho) d \rho$. Therefore we have

$$
\left|J_{l}^{ \pm}(|x|, \theta, \sigma, \varepsilon)\right| \leqslant \int_{-\infty}^{+\infty} Y\left( \pm\left\{<\theta, s^{l}(\theta)>|x|-\tau\right\}\right)|\tilde{\varphi}(\tau)| d \tau
$$

Thus we see that $J_{l}^{ \pm}(|x|, \theta, \sigma, \varepsilon)$ are bounded continuous functions of 
$(|x|, \theta, \sigma, \varepsilon)$ in $\left\{(|x|, \theta, \sigma, \varepsilon) ;|x|>0,|\theta|=1,\left|\sigma-\sigma^{0}\right|<\delta, 0 \leqslant \varepsilon<\varepsilon^{0}\right\}$. Further, we have

(21) $J_{l}^{ \pm}(|x|, \theta, \sigma, \varepsilon)=O\left(|x|^{-1}\right)$ uniformly in $\sigma$ and $\varepsilon$, as $|x| \rightarrow \infty$ for $\left\langle\theta, s^{l}(\theta)\right\rangle\left\langle 0\right.$ or $\left\langle\theta, s^{l}(\theta)\right\rangle>0$ according as " + " or " - ". If $\left\langle\theta, s^{l}(\theta)\right\rangle$ $\neq 0$, they have for $\varepsilon=0$ the forms

$$
\begin{aligned}
& J_{l}^{ \pm}(|x|, \theta, \sigma, 0) \\
& \quad= \pm i(2 \pi)^{(n-1) / 2} \exp \left\{i<\theta, s^{l}(\theta)>\sigma|x|\right\} \varphi(\sigma)+O\left(|x|^{-1}\right)
\end{aligned}
$$

as $|x| \rightarrow \infty$ for $\left\langle\theta, s^{l}(\theta)\right\rangle>0$ or $\left\langle\theta, s^{l}(\theta)\right\rangle\langle 0$ according as " + " or "-". On the other hand, we deduce from the estimate (11) that

$$
\varphi_{1}(\rho) q_{\nu}(\rho x)=O\left(|x|^{-(n+1) / 2}\right) \text { uniformly in } \rho \in\left(\sigma^{0}-\delta, \sigma^{0}+\delta\right)
$$

as $|x| \rightarrow \infty$ and that

$$
\left|\varphi_{1}(\rho) q_{\nu}(\rho x)-\varphi_{1}\left(\rho^{\prime}\right) q_{\nu}\left(\rho^{\prime} x\right)\right| \leqslant \text { const }|x|^{-(n+\alpha) / 2}\left|\rho-\rho^{\prime}\right|^{(1-\alpha) / 2}
$$

for all $\rho, \rho^{\prime} \in\left(\sigma^{0}-\delta, \sigma^{0}+\delta\right)$ as $|x| \rightarrow \infty . \quad(-1<\alpha<1)$.

Making use of the estimates (23), (24) we can show

$$
\int_{-\infty}^{+\infty} \frac{\varphi_{1}(\rho) q_{\nu}(\rho x)}{\rho-(\sigma \pm i \varepsilon)} d \rho=O\left(|x|^{-(n+\alpha) / 2}\right) \text { as }|x| \rightarrow \infty
$$

where the order relation is uniform in $\sigma \in\left(\sigma^{0}-\delta, \sigma^{0}+\delta\right)$ and in $\varepsilon \in\left[0, \varepsilon^{0}\right)$. Thus we have

Lemma 2. Let $\Phi(x ; \sigma \pm i \varepsilon)$ be the functions defined by (12). Then the following asymptotic formulas hold.

$$
\begin{aligned}
& \left(\frac{\partial}{\partial x}\right)^{\nu} \Phi(x ; \sigma \pm i \varepsilon)=\sum_{l} \exp \left\{i \frac{\pi}{4}\left(p_{l}^{+}(\theta)-p_{l}^{-}(\theta)\right)\right\} \\
& \quad \times \frac{\left(i s^{l}(\theta)\right)^{\nu} P\left(s^{l}(\theta)\right)}{\sqrt{\left|K\left(s^{l}(\theta)\right)\right| \cdot\left|\operatorname{grad} \lambda\left(s^{l}(\theta)\right)\right|}} J_{l}^{ \pm}(|x|, \theta, \sigma, \varepsilon)|x|^{-(n-1) / 2} \\
& \quad+O\left(|x|^{-n / 2}\right)
\end{aligned}
$$

where the summation is taken over $l$ such that $\left\langle\theta, s^{l}(\theta)\right\rangle \geqslant 0$ or that $\left\langle\theta, s^{l}(\theta)\right\rangle \leqslant 0$ according as $\sigma+i \varepsilon$ or $\sigma-i \varepsilon$.

In particular, if $\left\langle\theta, s^{l}(\theta)\right\rangle \neq 0$, 


$$
\begin{aligned}
& \left(\frac{\partial}{\partial x}\right)^{\nu} \Phi(x ; \sigma \pm i 0) \\
& = \pm i(2 \pi)^{(n-1) / 2} \sum_{l} \exp \left\{i\left\langle\theta, s^{l}(\theta)\right\rangle \sigma|x|+i \frac{\pi}{4}\left(p_{l}^{+}(\theta)-p_{l}^{-}(\theta)\right)\right\} \\
& \frac{\left(i s^{l}(\theta)\right)^{\nu} P\left(s^{l}(\theta)\right)}{\sqrt{\left|K\left(s^{l}(\theta)\right)\right|} \cdot\left|\operatorname{grad} \lambda\left(s^{l}(\theta)\right)\right|} \sigma^{d+|\nu|+(n-1) / 2}|x|^{-(n-1) / 2}+O\left(|x|^{-n / 2}\right) .
\end{aligned}
$$

In order to simplify the description of proof of our theorem, we now state some elementary facts as a lemma.

Lemma 3. Let $T_{\lambda}$ be a temperate distribution that depends on a parameter taking on values in a certain region $\Lambda$ of the complex plane $\boldsymbol{C}$, and assume that the Fourier transform $\hat{T}_{\lambda}(\xi)$ of $T_{\lambda}$ with respect to $x$ satisfies the conditions:

i) For every multi-index $\nu,\left(\frac{\partial}{\partial \xi}\right)^{\nu} \hat{T}_{\lambda}(\xi)$ is a continuous function of $(x, \lambda)$ in $g^{n} \times \Lambda$.

ii) For any fixed $\xi, \hat{T}_{\lambda}(\xi)$ is an analytic function of $\lambda$.

iii) For every $\nu$, there exists a constant $C_{\nu}$ such that

$$
\left|\left(\frac{\partial}{\partial \xi}\right)^{\nu} \hat{T}_{\lambda}(\xi)\right| \leqslant C_{\nu}(1+|\xi|)^{r-|\nu|}
$$

where $C_{\nu}$ does not depend on $\xi$ and on $\lambda$.

Then $T_{\lambda}$ has the following properties.

$1^{\circ} \quad$ If $|\nu| \geqslant n+r+1+|\beta|,\left(\frac{\partial}{\partial x}\right)^{\beta}\left(x^{\nu} T_{\lambda}\right)$ is a continuous function of $(x, \lambda)$ in $\boldsymbol{R}^{n} \times \Lambda$ and analytic in $\Lambda$ with respect to $\lambda$.

$2^{\circ}$ For every $\beta,\left(\frac{\partial}{\partial x}\right)^{\beta} T_{\lambda}$ is a continuous function of $(x, \lambda)$ in $\left(\boldsymbol{R}^{n}-\{0\}\right) \times \Lambda$ and analytic in $\Lambda$ with respect to $\lambda$. Further, as $|x| \rightarrow \infty$, $\left(\frac{\partial}{\partial x}\right)^{\beta} T_{\lambda}$ converges to zero more rapidly than any power of $1 /|x|$, where the convergence is uniform with respect to $\lambda$ in $\Lambda$.

\section{Proof of the Theorem}

First note that because of the assumption i), the roots $\lambda_{k}(\xi), k=1, \ldots$, $m$ of $\Delta(\xi, \lambda)=0$ are analytic function of $\xi$ in $\boldsymbol{g}^{n}-\{0\}$ and positively 
homogeneous of degree 1. Further we have from convention (4)

$$
\lambda_{k}(-\xi)=-\lambda_{m-k+1}(\xi) \text { and } \alpha_{k}=\alpha_{m-k+1}, k=1, \ldots, m .
$$

Hence, by the assumption ii) we have for $\xi \neq 0$

$$
\begin{gathered}
\lambda_{1}(\xi)>\cdots>\lambda_{m / 2}(\xi)>0>\lambda_{m / 2+1}(\xi)=-\lambda_{m / 2}(-\xi)>\cdots \\
\cdots>\lambda_{m}(\xi)=-\lambda_{1}(-\xi) \quad \text { if } m \text { is even }
\end{gathered}
$$

and

$$
\begin{gathered}
\lambda_{1}(\xi)>\cdots>\lambda_{[m / 2]}(\xi)>\lambda_{[m / 2]+1}(\xi) \equiv 0>\lambda_{[m / 2]+2}(\xi)=-\lambda_{[m / 2]}(-\xi)> \\
\cdots>\lambda_{m}(\xi)=-\lambda_{1}(-\xi) \quad \text { if } m \text { is odd. }
\end{gathered}
$$

We denote by $P_{k}(\xi)$ the projection matrix in $\mathbb{C}^{N}$ onto the eigenspace of $A(\xi)$ corresponding to the eigenvalue $\lambda_{k}(\xi)$. As is well-known, $P_{k}(\xi)$ can be represented in the form.

$$
\begin{aligned}
P_{k}(\xi) & =\frac{1}{2 \pi i} \int_{\Gamma_{k}(\xi)}(\lambda I-A(\xi))^{-1} d \lambda \\
& =\frac{1}{\left(\alpha_{k}-1\right) !}\left(\frac{d}{d \lambda}\right)^{\alpha_{k}-1}\left\{\frac{\operatorname{cof}(\lambda I-A(\xi))}{\frac{\operatorname{det}(\lambda I-A(\xi))}{\left(\lambda-\lambda_{k}(\xi)\right)^{\alpha_{k}}}}\right\}_{\lambda=\lambda_{k}(\xi)}
\end{aligned}
$$

where $\Gamma_{k}(\xi)$ is a contour enclosing a domain that contains the point $\lambda_{k}(\xi)$ and does not contain other points of the spectrum of $A(\xi)$. Further they enjoy the properties:

$$
\begin{aligned}
& P_{1}(\xi)+\cdots+P_{m}(\xi)=I \\
& P_{j}(\xi) P_{k}(\xi)=\delta_{j k} P_{j}(\xi), j, k=1, \cdots, m .
\end{aligned}
$$

Using the $P_{k}, k=1, \ldots, m$, we can represent $(A(\xi)-\lambda I)^{-1}$ for any non-real $\lambda$ as follows:

$$
(A(\xi)-\lambda I)^{-1}=\sum_{k=1}^{m}\left(\lambda_{k}(\xi)-\lambda\right)^{-1} P_{k}(\xi)
$$

For brevity we will consider only the case: $m$ is odd, $\lambda$ varies in $\Lambda^{+}$ $=\left\{\lambda=\sigma+i \varepsilon ;\left|\sigma-\sigma^{0}\right|<\delta, 0 \leqslant \varepsilon<\varepsilon^{0}\right\}, \sigma^{0}>0$ and $\nu=0$, since the other case can be proved with obvious modifications of the present one. 
Now let us consider the $[m / 2]$ disjoint closed surfaces defined by $S_{k}=\left\{s ; \lambda_{k}(s)=1, s \in g^{n}\right\}, k=1, \ldots,[m / 2] \equiv b$. They enclose the origin, and $S_{1}$ defines the innermost sheet, $S_{2}$ defines the next, etc. and $S_{b}$ defines the outermost sheet. Choose relatively compact open subsets $U_{k}$ and $V_{k}, k=0,1, \ldots,[m / 2]=b$ of $g^{n}$ which satisfy the conditions:

i) $U_{0}$ and $V_{0}$ are neighborhoods of the origin, and $U_{k}, V_{k}$ are neighborhoods of $\sigma^{0} S_{k}$ for each $k(1 \leqslant k \leqslant b)$. ii) $\bar{U}_{k} \subset V_{k}, k=0,1, \ldots, b$. iii) $V_{0}, \ldots, V_{b}$ are disjoint each other. Let $\phi_{k} \in C_{0}^{\infty}\left(V_{k}\right)$ and satisfy $\phi_{k}(\xi)=1$ on $U_{k}$. With the aid of the functions $\phi_{k}$, we can write $E(x: \lambda)$ as follows:

$$
\begin{aligned}
& E(x, \lambda)=\mathscr{F}^{-1}\left[\sum_{k=1}^{b} \phi_{k}(\xi)\left(\lambda_{k}(\xi)-\lambda\right)^{-1} P_{k}(\xi)\right] \\
& \quad+\mathscr{F}^{-1}\left[\phi_{0}(\xi)(A(\xi)-\lambda I)^{-1}\right]-\frac{1}{\lambda} \mathscr{F}^{-1}\left[\phi_{b+1}(\xi) P_{b+1}(\xi)\right] \\
& \quad+\mathscr{F}^{-1}\left[\sum_{k=1}^{b}\left(1-\phi_{0}(\xi)\right)\left(1-\phi_{k}(\xi)\right)\left(\lambda_{k}(\xi)-\lambda\right)^{-1} P_{k}(\xi)\right. \\
& \left.\quad+\sum_{k=b+2}^{m}\left(1-\phi_{0}(\xi)\right)\left(\lambda_{k}(\xi)-\lambda\right)^{-1} P_{k}(\xi)\right] \equiv \sum_{j=1}^{4} I_{j}(x ; \lambda), \text { respectively, }
\end{aligned}
$$

where $\mathscr{F}^{-1}$ denotes the inverse Fourier transformation.

Consider first $I_{2}(x ; \lambda)$ : We take $\delta>0$ properly small. Then if $\lambda \in \Lambda$ $=\left\{\lambda ;\left|\operatorname{Re} \lambda-\sigma^{0}\right|<2 \delta\right\}, \phi_{0}(\xi)(A(\xi)-\lambda I)^{-1}$ is a $C^{\infty}$ function of $\xi$ with compact support, and analytic in $\lambda$. Hence $I_{2}(x ; \lambda)$ is a $C^{\infty}$ function of $x$ and analytic in $\lambda$. Further, for any $\nu$ and any integer $p \geqslant 0$, we have

$$
\begin{array}{r}
\left(\frac{\partial}{\partial x}\right)^{\nu} I_{2}(x ; \lambda)=O\left(|x|^{-p}\right) \text { and }\left(\frac{\partial}{\partial \lambda}\right)\left(\frac{\partial}{\partial x}\right)^{\nu} I_{2}(x ; \lambda)=O\left(|x|^{-p}\right) \\
\text { for }|x| \rightarrow \infty
\end{array}
$$

where the order relation is uniform (with respect to $\lambda$ ) on compact subsets of $\Lambda$.

Consider next $I_{4}(x ; \lambda)$ : The function in brackets satisfies the condition (27) of Lemma 3. Therefore $I_{4}(x ; \lambda)$ is a $C^{\infty}$ function of $x$ in $\boldsymbol{R}^{n}-\{0\}$, and analytic in $\lambda \in \Lambda$. Moreover, for any integer $p \geqslant 0$

$$
\left(\frac{\partial}{\partial x}\right)^{\nu} I_{4}(x ; \lambda)=O\left(|x|^{-p}\right) \text { and }\left(\frac{\partial}{\partial \lambda}\right)\left(\frac{\partial}{\partial x}\right)^{\nu} I_{4}(x ; \lambda)=O\left(|x|^{-p}\right)
$$


as $|x| \rightarrow \infty$. $I_{3}(x ; \lambda)$ possesses also the same properties.

We now return to $I_{1}(x ; \lambda)$. If $\left|\sigma-\sigma^{0}\right|<2 \delta$ and $0<\varepsilon<\varepsilon^{0}, I_{1}(x ; \lambda)$ is represented in the integral form:

$$
\begin{aligned}
I_{1}(x ; \sigma+i \varepsilon) & =(2 \pi)^{-n} \sum_{k=1}^{b} \int \frac{\exp \{i<x, \xi>\} P_{k}(\xi)}{\lambda_{k}(\xi)-(\sigma+i \varepsilon)} \phi_{k}(\xi) d \xi \\
& \equiv(2 \pi)^{-n} \sum_{k=1}^{b} \Phi_{k}(x ; \sigma+i \varepsilon) .
\end{aligned}
$$

Thus $I_{1}(x ; \sigma+i \varepsilon)$ is a sum of integrals of the type (12). By applying Lemma 2 to each $\Phi_{k}(x ; \sigma+i \varepsilon)$ and putting together the above discussion, we see that $E(x ; \lambda)$ has the properties $2^{\circ}$ and $3^{\circ}$ of the theorem. The property $4^{\circ}$ for $E(x ; \lambda)$ can be shown in the same way in $[6]$. From (25), (26) and the formula:

$$
\left(\frac{\partial}{\partial x}\right)^{\nu} E(x ; \sigma \pm i \varepsilon)=(2 \pi)^{-n} \sum_{k=1}^{b}\left(\frac{\partial}{\partial x}\right)^{\nu} \Phi_{k}(x ; \sigma \pm i \varepsilon)+O\left(|x|^{-p}\right)
$$

as $|x| \rightarrow \infty$ for any integer $p \geqslant 0$, it is easy to find the explicit forms of $e_{\nu}^{ \pm}(x, \sigma, \varepsilon)$ in (7) of the theorem.

In fact, by applying Lemma 2 to each $\Phi_{k}$, we obtain for $\sigma \in\left(\sigma^{0}-\delta\right.$, $\left.\sigma^{0}+\delta\right)$ and $\varepsilon \in\left[0, \varepsilon^{0}\right)$

$$
\begin{aligned}
& e_{\nu}^{ \pm}(x, \sigma, \varepsilon)=(2 \pi)^{-n} \sum_{k=1}^{b} \sum_{l_{k}} \exp \left\{i \frac{\pi}{4}\left(p_{k, l_{k}}^{+}(\theta)-p_{k, l_{k}}^{-}(\theta)\right)\right\} \\
& \times \frac{\left(i s^{k, l_{k}}(\theta)\right)^{\nu} P\left(s^{k, l_{k}}(\theta)\right)}{\sqrt{\left|K\left(s^{k, l_{k}}(\theta)\right)\right|}\left|\operatorname{grad} \lambda_{k}\left(s^{k, l_{k}}(\theta)\right)\right|} J_{k, l_{k}}^{ \pm}(|x|, \theta, \sigma, \varepsilon) .
\end{aligned}
$$

Here the summation $\sum_{l_{k}}$ is taken over $l_{k}$ such that $\left\langle\theta, s^{k, l_{k}}(\theta)\right\rangle \geqslant 0$ or that $\left\langle\theta, s^{k, l_{k}}(\theta)\right\rangle \leqslant 0$ according as $\sigma+i \varepsilon$ or $\sigma-i \varepsilon$, and

$$
\begin{aligned}
& \text { (39) } J_{k, l_{k}}^{+}(|x|, \theta, \sigma, \varepsilon) \\
& =(2 \pi)^{(n+1) / 2} \int_{-\infty}^{<\theta, s^{k, l_{k}(\theta)>|x|}} \tilde{\varphi}(\tau) \exp \left\{i(\sigma+i \varepsilon)\left(<\theta, s^{k, l_{k}}(\theta)>|x|-\tau\right)\right\} d \tau, \\
& (39)^{\prime} \quad J_{\vec{k} l_{k}}(|x|, \theta, \sigma, \varepsilon) \\
& =-(2 \pi)^{(n+1) / 2} \int_{<\theta, s^{k, l_{k}(\theta)>|x|}}^{+\infty} \tilde{\varphi}(\tau) \exp \left\{i(\sigma-i \varepsilon)\left(<\theta, s^{k, l_{k}}(\theta)>|x|-\tau\right)\right\} d \tau
\end{aligned}
$$


where

$$
\tilde{\varphi}(\tau)=\int_{-\infty}^{+\infty} \exp \{i \rho \tau\} \varphi(\rho) d \rho
$$

If $\left\langle\theta, s^{k, l_{k}}(\theta)\right\rangle \neq 0$ they have for $\varepsilon=0$ the form

$$
\begin{aligned}
J_{k, l_{k}}^{ \pm} & (|x|, \theta, \sigma, 0) \\
= & \pm i(2 \pi)^{(n-1) / 2} \sigma^{(n-1) / 2+|\nu|} \exp \left\{i<\theta, s^{k, l_{k}}(\theta)>\sigma|x|\right\} \\
& +O\left(|x|^{-1}\right)
\end{aligned}
$$

as $|x| \rightarrow \infty$ for $\left\langle\theta, s^{k, l_{k}}(\theta)\right\rangle>0$ or $\left\langle\theta, s^{k, l_{k}}(\theta)\right\rangle\langle 0$ according as " + " or "-.". We can also obtain for $\sigma<0$ the corresponding formulas of $e_{\nu}^{ \pm}(x, \sigma, \varepsilon)$ in the same way.

\section{References}

[1] Grušin, V. V., On Sommerfeld-type conditions for a certain class of partial differential equations, Mat. Sb. (N.S.) 61 (103) (1963), 147-174. A.M.S. Transl. Ser. 2, 51 (1966), 82-112.

[2] Littman, W., Fourier transforms of surface-carried measures and differentiability of surface averages, Bull, Amer. Math. Soc. 69 (1963), 766-770.

[3] — Decay at infinity of solutions of partial differential equations with constant coefficients, Trans. Amer. Math. Soc. 123 (1966), 449-459.

[4] Matsumura, M., Comportement des solutions de quelques problèmes mixtes pour certains systèmes hyperboliques symétriques à coefficients constants, Publ. RIMS, Kyoto Univ. Ser. A, 4 (1968), 309-359.

[5] - Asymptotic behavior of solutions of mixed problems for symmetric hyperbolic systems of 1st order with constant coefficients, Proc. of the Symposium on Functional Analysis and Related Topics held at R. I. M. S. Kyoto Univ., 59 (Dec. 1968), 140-172 (Japanese).

[6] Mochizuki, K., Spectral and scattering theory for symmetric hyperbolic systems in an exterior domain, Publ. RIMS, Kyoto Univ. 5 (1969), 219-258.

[7] Schulenberger, J.R., The Green's matrix for steady-state wave propagation in a class of inhomogeneous anisotropic media, Arch. Rational Mech. Anal. 34 (1969), 380-402.

[8] Vainberg, B.R., Principle of radiation, limit absorption and limit amplitude in the general theory of partial differential equations, Usp. Mat. Nauk 21 (1966), 115-194.

[9] Wilcox, C.H., Steady-state wave propagation in homogeneous anisotropic media, Arch. Rational Mech. Anal. 25 (1967), 201-242. 
